\title{
Germanica
}

\section{Métaphysique et humanisme chez Platon}

(note sur la lecture de Platon par Heidegger)

Metaphysik und Humanismus bei Plato: Wie Heidegger Plato verstand

\section{Lucien Bescond}

\section{CpenEdition}

Journals

Édition électronique

URL : http://journals.openedition.org/germanica/2438

DOI : 10.4000/germanica.2438

ISSN : 2107-0784

Éditeur

Université de Lille

\section{Édition imprimée}

Date de publication : 31 décembre 1990

Pagination : 143-150

ISBN : 9782913857025

ISSN : 0984-2632

\section{Référence électronique}

Lucien Bescond, « Métaphysique et humanisme chez Platon », Germanica [En ligne], 8 | 1990, mis en ligne le 28 novembre 2014, consulté le 06 octobre 2020. URL : http://journals.openedition.org/ germanica/2438; DOI : https://doi.org/10.4000/germanica.2438

Ce document a été généré automatiquement le 6 octobre 2020.

(C) Tous droits réservés 


\title{
Métaphysique et humanisme chez Platon
}

\author{
(note sur la lecture de Platon par Heidegger) \\ Metaphysik und Humanismus bei Plato: Wie Heidegger Plato verstand
}

\section{Lucien Bescond}

1 Avec Platon, selon Heidegger, «l'essence de la vérité » reçoit la détermination d'être «l'exactitude de la représentation qui s'énonce». La vérité devient l'objet d'une énonciation discursive, accordée à la représentation de l'esprit : la vérité est l'objet du logos, lui-même fonction de la science dialectique. Le discours sur la vérité se donne comme critère l'exactitude de la visée de son objet. Cet idéal d'exactitude donne le style de la détermination de la vérité chez Platon et pour l'histoire ultérieure de la métaphysique.

Du poids historique de la détermination platonicienne, Heidegger prend trois exemples - chez Thomas d'Aquin, Descartes et Nietzsche - pour en montrer, sous des langages différents, la répétition de la même préoccupation, initialement advenue avec Platon.

Selon Heidegger, Thomas d'Aquin détermine, à la suite de Platon, l'entendement, humain ou divin, comme le lieu de la vérité ; c'est dans un entendement (intellectus) que la vérité se donne, ou qu'elle se représente. Alors, la vérité (comme "veritas", «Wahrheit») ne peut plus être "alètheia », au sens antéplatonicien, pré-socratique, au sens du dévoilement de l'être comme tel; la vérité devient "adaequatio", ressemblance et conformité, de ce qui se donne dans l'exactitude aux canons de la représentation en un entendement. Platon opère une révolution dans la détermination de la vérité : il passe de l'époque où elle est dévoilement de l'être à l'époque où elle devient valeur d'exactitude dans les conditions d'une représentation d'entendement.

Avec Descartes, l'idée se renforce que l'intellect seul est le lieu de la vérité ou de la fausseté, car la vérité est de l'ordre d'une proposition et elle en constitue un caractère : elle est donc une marque de la représentation que l'entendement se fait d'une chose, non un apparaître propre à la chose elle-même. 
Quant à Nietzsche, au terme du plein accomplissement du destin de la métaphysique inauguré par Platon, il conjugue la vérité à la valeur («Wert») opératoire qu'elle peut comporter dans le plan de développement et d'expression d'une vie ; alors, même en étant une "sorte d'erreur", la vérité demeure vraie en servant l'expression de cette vie. Mais alors, elle n'est plus "qu'un trait de pensée »: une manière pour un vivant d'affronter le réel («das Wirkliche») en le représentant. La vérité est de l'ordre d'une parade du vivant pour construire, à son profit, une "soi-disant réalité ", qui même trompeuse et inexacte, lui permette de survivre.

6 Donc, même quand Nietzsche renverse en son contraire la détermination platonicienne de la vérité comme " exactitude de renonciation ", il demeure fidèle, pour Heidegger, à la conception traditionnelle depuis Platon: celle, une fois encore, de fixer la vérité comme valeur d'exactitude de la représentation; même comprise comme « une sorte d'erreur ", la vérité est encore valeur de la représentation de l'homme confronté à l'effectivité de la vie et forcé d'avoir à ériger contre le flux du devenir («Werden») les normes soi-disant vraies de sa propre vérité.

7 Ainsi, de Platon à Nietzsche, une même période de la détermination de la vérité développe les conséquences de la postulation originaire accomplie par Platon, en rupture avec les positions antérieures des penseurs présocratiques. Là où Nietzsche finit par dire que la vérité est une "sorte d'erreur", il demeure encore dans la mouvance extrême de la position platonicienne.

La césure majeure c'est donc la transformation par Platon de la détermination présocratique, où la vérité n'était encore que «le non-voilement de l'étant», en sa propre thèse de la vérité comme " exactitude du regard ». Le penseur présocratique se met en situation d'accueillir l'être (en tant qu'il se donne dans «le non-voilement de l'étant »). Platon, lui, situe et détermine ce qui se donne de l'être dans l'étant selon le regard qu'il prend sur lui ; ce regard dont parle Heidegger n'est ici que la conversion qu'il effectue de la "théorie" platonicienne, la vue que peut se donner l'intellect humain des Formes et des Idées, qui se présentent à lui. En somme, la pensée présocratique aborde, sans le déterminer, ce qui se donne à l'homme de l'être, quand la philosophie platonicienne (et à partir d'elle, toute la tradition de la métaphysique) aborde l'être de l'étant selon une détermination intelligible. Ici, Heidegger choisit, dans le complexe vocabulaire de Platon, le terme d'«idéa», l'Idée ou la Forme. Elle sera désormais le critère de la détermination, quant à l'intellect humain, de ce qui se donne de l'être à l'investigation qu'il en poursuit. Il s'agit donc "d'une interprétation de l'étant » («Auslegung des Seienden»). La philosophie, comme méta-physique, porte une interprétation à l'égard de l'étant. Cette interprétation abandonne la figure présocratique de la vérité comme "dévoilement " ("Entbergung»), venue «à la présence ». Avec Platon, ce qu'il en est de l'être comme "arrivée-à-la présence » se confondra désormais avec son "idéa", sa forme intelligible. Qu'en est-il alors de son statut?

9 Heidegger fait remarquer que la forme «n'est pas subordonnée au non-voilement ». On peut comprendre que "l'idéa » n'est pas un pur apparaître, qui aurait perdu tout rapport avec ce qui demeure caché et voilé derrière lui. Le statut de «l'idéa » comme apparaître est complexe, car deux plans peuvent s'observer : le premier, celui du « fait de paraître »; le second, intérieur « à l'essence du paraître », la condition de fond de la manifestation qui se produit par "l'idéa", quand elle vient à se montrer. S'il faut distinguer deux degrés de l'apparaitre, « l'idéa » ne peut être tenue pour « un premier 
plan de Paletheia »; puisque le paraitre s'est dédoublé en deux plans, une apparence et une condition de l'apparence, saisir l'apparence - ce ne peut être que dans une "idéa " - ce n'est pas s'approcher du "premier plan de Paletheia ». La vérité s'éloigne, alors que l'effort de la pensée présocratique (Héraclite et Parménide) consistait, au contraire, à s'en approcher. Mais, Heidegger concède à la doctrine platonicienne de "l'idéa " qu'elle garde quelque sorte de rapport à la vérité ; c'est elle qui fonde la possibilité de l'apparaître, bien que, désormais, l'être propre de la vérité demeurera "inconnu ", inaccessible aux prises d'une pensée qui s'est repliée sur l'appréhension de l'idée. Alors, Heidegger peut déduire que "la vérité n'est plus, comme non-voilement le trait fondamental de l'être lui-même ", ce qu'elle était, justement, à l'époque de l'approche présocratique. La vérité est devenue autre du fait de l'interprétation philosophique de Platon : elle est - et demeurera - «exactitude » dans la conformité à l'idée, dans la sphère d'un apparaître, où la pensée vient à se repérer et à s'orienter, dans la mesure où elle produit «la connaissance de l'étant». Ce qui est gagné en objectivité et en exactitude est pour autant perdu du côté de la relation libre du penser à la vérité, comme trait propre de l'Être lui-même. L'idéal de la connaissance philosophique devient celui « d'une connaissance rigoureuse », pourrait-on dire avec Husserl.

Heidegger combine à ses remarques sur la visée de la vérité son interprétation de la doctrine platonicienne de la culture (" paideia»). Heidegger, en effet, commente le passage de la "République », où Platon expose l'allégorie de la Caverne (qu'Heidegger comprend comme une histoire, "Geschichte »). Il s'agit de montrer que vont de pair la doctrine de la vérité et la thèse, figurée symboliquement par la Caverne, de la possibilité pour les hommes d'accéder à l'éducation, en effectuant la remontée du séjour ombreux au jour lumineux de l'extérieur. L'allégorie de la caverne n'est donc pas seulement une image qui n'aurait pour objet que l'éducation (signalons ici que le terme de "paideia " est présent, dès le début du texte, de même que son contraire, " apaideusia ", l'inculture). L'histoire décrit le processus de la conversion éducative et celui-là même, analysé plus haut, de la conversion de l'attitude philosophique par rapport à l'étant. Dans les deux cas, il y a passage («Ubergang ») : et, dans la Caverne, passage " d'un lieu de séjour à l'autre ». Que faut-il entendre par là?

11 La différence des deux lieux (l'intérieur et l'extérieur de la caverne) ne vaut pas seulement du prisonnier, progressivement libéré de ses entraves : elle vaut aussi de "l'être de la sophia», qui change d'une appréhension indistincte des ombres à l'intérieur à une appréhension progressivement plus claire des réalités hors de la caverne. Donc, la remontée vers la clarté pour le prisonnier symboliserait, selon Heidegger, la progression même de la sagesse et du savoir par la pratique de la philosophie.

12 Heidegger définit la «sophia » (sagesse pratique ou théorique, sagesse du « sophiste » aussi) comme la compétence et la capacité de décision: en un premier aspect, compétence du spécialiste. Mais elle est aussi aptitude à saisir (pour le philosophe dialecticien, selon Platon) "le non-voilé », ce qui se donne, on l'a vu, dans l'apparaître de «l'idéa ». Elle est, par là, faculté d'identifier telle «idéa », en la différenciant d'une autre. Pour le prisonnier de la caverne et ses compagnons, ce qui vaut, c'est le pouvoir de reconnaître les ombres qui défilent sur la paroi. Si elle sera désignée, par le prisonnier délivré, «la sagesse de là-bas », elle n'en forme pas moins une sophia, un pouvoir de s'y reconnaître dans la défilement des ombres. 
Une fois sorti de la caverne, une autre " sophia » est possible qui « surpasse et domine » la «sophia » de l'intérieur de la caverne. La «sophia », nouvelle pour le prisonnier délivré, et supérieure à la première, revient à la « sophia » de la philosophie : visée de l'être de l'étant dans le milieu des "Idées » et sous la caution des Idées. Si la "sophia " se contente, dans la caverne, de l'appréhension des apparences projetées, la "sophia " supérieure "dépasse les choses immédiatement présentes " - dans leur apparence sensible - pour tenter d'appréhender, en s'y repérant, « le Permanent ». La "sophia » supérieure veut la saisie de la permanence des Idées, supposées se montrer par ellesmêmes. À ce degré, un ordre de manifestation est donné, supérieur à celui de la manifestation dans l'apparence sensible. « sophia » supérieure : attirance, prédilection et amitié ("philia ») qui peuvent rendre raison d'un désir de connaître supérieur à la simple reconnaissance de l'apparence sensible. La " philosophia » est justement l'élan vers ce type de sagesse, et la remontée du prisonnier symbolise le mouvement de ce dépassement de la «sophia» de l'intérieur de la caverne, qui ne vaut que comme reconnaissance des apparences en mouvement. Ainsi donc, « hors de la caverne », « la sophia est philosophia ». C'est parce que du sein de l'étant, où elle demeure située, la pensée s'élance vers la recherche des Idées, qu'elle peut être comprise comme philosophie, amour de la captation d'une sagesse qui fera advenir, en la reconnaissant, l'Idée inhérente à l'être de l'étant. Le mouvement de la philosophie ne peut être, ici, que méta-physique. familier. Il la conduit à partir de la préposition grecque «méta " (qui joue le rôle de préfixe dans la formation savante du terme de méta-physique), dont il repère un usage significatif dans la lettre du texte de Platon. Il s'agit du passage où Socrate explique à son interlocuteur Glaucon que le prisonnier délivré finira par saisir que le soleil "gouverne tout dans le monde visible et qu'il est en quelque manière la cause de toutes ces choses que lui et ses compagnons voyaient dans la caverne ». Ce à quoi Glaucon réplique : « il est évident que c'est là qu'il en viendrait après ces diverses expériences ». C'est justement ici, sur cet "après ", qu'Heidegger fait porter son commentaire. La saisie par le prisonnier du soleil comme cause ordonnatrice est postérieure à l'expérience de sa conversion, mais elle implique encore un dépassement et le sens d'une transcendance. Le prisonnier a dépassé et transcendé son expérience première, celle de l'appréhension sensible des ombres dans la caverne ; il est passé d'un plan à un autre, d'une "Sophia » à une autre, d'un mode de visée sensible à la visée théorique des Idées. «Ce regard non-sensible » vise l'idée comme le supra-sensible : Heidegger ajoute ici que la région supra-sensible est organisée dans la dépendance à une « Idée de toutes les autres Idées ", l'Idée du Bien, raison et cause de tout de ce qui existe dans le monde intelligible. «L'étant » est donc non point ce qui s'offre à l'intuition sensible et à la vision des yeux, mais ce qui se donne, parce qu'intelligible, à la pensée et à l'appréhension intuitive de la "sophia " philosophique. "L'être de l'étant », ce en quoi il demeure, repose, a consistance et permanence, est « Idée ». Et, à son tour, l'Idée, ou plutôt les Idées, tiennent leur être et leur existence d'une Idée qui les fonde, tout en les dépassant, Idée des autres Idées, l'Idée du Bien.

Heidegger note ensuite l'extension que donnera Aristote à la pensée de Platon : car le «Bien » va devenir le divin, celui de la pensée qui se pense elle-même. Ce qui permet alors à Heidegger d'avancer en une formule synthétique: «depuis que l'être a été 
interprété comme idéa, la pensée ("Denken") tournée vers l'être de l'étant est métaphysique, et la métaphysique est théologie ». On peut comprendre qu'Heidegger cumule ici les résultats des démarches précédentes: Platon a orienté toute la recherche philosophique en postulant le caractère idéel de l'être de l'étant; mais ce postulat impliquait la transcendance méta-physique; la pensée, pour penser l'Idée, doit métaphysiquement dépasser l'étant sensible (donné à l'intuition des sens) vers le lieu supra-sensible. Par là, avec Platon et avec Aristote, la philosophie est devenue métaphysique (ce qu'elle n'était pas chez les présocratiques); la forme de cette métaphysique est discours logique sur l'être de l'étant, soit onto-logie.

La suite de ces transformations est la conséquence d'un événement initial, le changement dans "l'essence de la vérité », opéré par Platon, alors que pour la pensée présocratique, l'être demeure, proche et éloigné de la pensée. Avec Platon, par lui et après lui, l'être est visé comme «idéa ». Dès lors, la visée de "l'idéa » devient d'une "excellence spéciale». Ce regard métaphysique et cette visée supra-sensible deviennent la valeur qui oriente la méthode philosophique et conditionnent la « remontée » hors de la caverne (ce que Mallarmé, poétiquement, aura mieux dit que tout commentaire exégétique : « Gloire du long désir, Idées »).

Mais alors aussi l'excellence de l'être idéel à viser retentit sur la méthode de formation de l'individu, sur la «paideia». Former l'individu sera le préparer à accomplir le dépassement par la pensée, vers l'idéal de la visée du supra-sensible. Mais aussi, la formation à la "méta-physique » attire l'intérêt sur la "position » de l'homme et sa situation par rapport à la visée (possible et envisageable selon une formation et un programme d'éducation). L'orientation de l'homme devient déterminante; c'est en formant - et, éventuellement, en rectifiant - cette orientation que l'homme pourra accéder à la visée des Idées dans la région du supra-sensible.

On peut donc saisir la proposition d'Heidegger que le début de la méta-physique, avec Platon, soit aussi le début de "l'humanisme" dans la philosophie. Par ce terme, Heidegger entend, au sens le plus général, toute position de principe qui assigne à l'homme (et à l'humanité) une position « centrale » dans l'étant. L'homme est centre d'une perspective : l'intérêt de l'homme (son intérêt spéculatif comme pratique) est la valeur d'orientation pour la philosophie. Il s'agit de poser le privilège de la position et des intérêts de l'homme ; et le paradoxe, qui n'est que d'apparence, est que le premier à accomplir rigoureusement cette position de l'humanisme soit Platon, dont la métaphysique semble chercher à "dépasser » le plan des contingences humaines, en fuyant vers le suprasensible. Mais, ce déplacement des intérêts immédiats (tels ceux des hommes enfermés dans la caverne) est conduit vers une visée métaphysique : cette visée est à même de procurer une attention supérieure à la culture des intérêts humains véritables. Le prisonnier qui sort de la caverne devra revenir vers ses compagnons pour leur enseigner, autant qu'il se peut, à conduire leur vie en fonction de la connaissance, qu'il aura pu obtenir, des Idées. L'homme n'est donc pas encore « l'étant » suprême (celui que finira par proclamer Nietzsche), puisqu'il est dépassé par une Mesure supérieure à lui. Mais qu'il puisse viser cette Mesure ou cette Norme lui donne la possibilité de constituer son existence avec certitude. Le dogmatisme métaphysique engendre un corrélat nécessaire, la position humaniste.

Avec Platon, selon Heidegger, est donc en question et le début de la métaphysique et celui de la vision humaniste. Humanisme et métaphysique vont donc de pair, avec et depuis Platon. 


\section{RÉSUMÉS}

Heidegger est suivi, ici, dans certains des passages les plus significatifs de son exposé sur la doctrine de Platon sur la vérité («Plato's Lehre von der Wahreit»). On cherche à montrer la direction de la méthode qu'utilisait Heidegger dans ce texte célèbre : méthode de lecture et aussi méthode d'interprétation de la démarche de Platon (notamment, celle qu'il suivait dans le mythe de la Caverne du dialogue "la République »). On cherche aussi à méditer les résultats de la lecture de Platon par Heidegger; ces résultats sont importants; en effet, Platon est compris comme celui qui met fin au style du penser présocratique; il est aussi entendu comme le novateur qui déterminera le destin de la méta-physique occidentale jusqu'à nous.

Es werden in diesem Beitrag einige der prägnantesten Auszüge von Heideggers Darstellung der Lehre Piatos über die Wahrheit (Piatos Lehre von der Wahrheit) analysiert. Dabei wird der Akzent auf das methodische Verfahren gelegt, das Heidegger in seinem bekannten Text anwendet: sowohl was seine Lektüre als auch seine Interpretierung von Piatos Gedankengang betrifft (besonders in Bezug auf den Höhlenmythos im Dialog Die Republik). Ebenso werden die Schlussfolgerungen, die Heidegger aus seiner Lektüre Piatos zieht, nachhaltig betrachtet. Plato erscheint hier als derjenige, der den Schlußstrich zieht unter die vorsokratische Denkweise. Er wird auch als der Erneuerer aufgefasst, der das Schicksal der abendländischen Metaphysik bis in unsere Zeit hinein bestimmen wird.

\section{AUTEUR}

\section{LUCIEN BESCOND}

Université de Lille III 